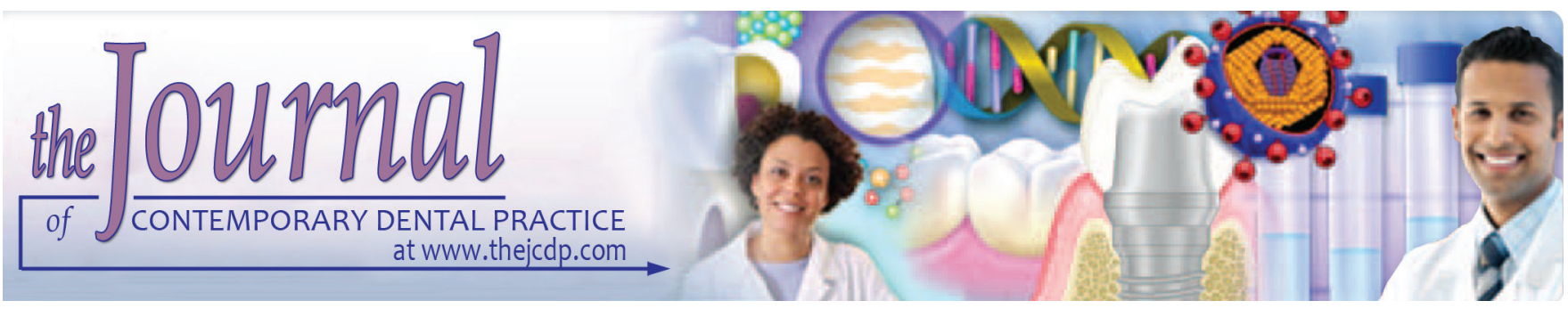

\title{
Discoloration Effects of Traditional Turkish Beverages on different Composite Restoratives
}

\author{
${ }^{1}$ Serdar Baglar, ${ }^{2}$ Erol Keskin, ${ }^{3}$ Tahir Orun, ${ }^{4}$ Abdulhamit Es
}

\begin{abstract}
Aim: The aim of this study was to evaluate the discoloring effects of five beverages including, especially, traditional Turkish ones on five commonly used dental composites by using a spectrophotometer device.

Materials and methods: Five methacrylate-based composites (shade A2) were selected to evaluate their color stability (175 disk samples). Four of them (Filtek Ultimate Universal, Clearfil Majesty ES-2, Tetric EvoCeram, and Cavex Quadrant Universal LC) were nanofilled universal composites for both anterior and posterior restorations, and one (Clearfil Majesty Posterior) was nano-superfilled for posterior restorations. The tested beverages were tamarind syrup, ottoman syrup, turnip juice, pomegranate juice, and distilled water (control). All samples were kept in an incubator at $37^{\circ} \mathrm{C}$ for 12 days (measured at 3rd, 6th, 12th day intervals) in immersion solutions which was equivalent to 1 year in vivo. Color measurements were made with VITA Easyshade Advance (Vident, Brea, CA) spectrophotometer device according to CIE L*a*b* system. Statistical analysis was performed with analysis of variance and least significant difference test to analyze differences in $L^{*} a^{*} b^{*}$ and $\Delta E$ values.
\end{abstract}

Results: All materials showed significant discoloration $(p<0.05)$ when compared with the control group. The highest $\Delta \mathrm{E}$ was observed in turnip juice, whereas ottoman syrup had the lowest $\Delta \mathrm{E}$. Tetric EvoCeram showed the lowest $\Delta \mathrm{E}$, while Clearfil Majesty ES-2 showed the highest $\Delta \mathrm{E}$.

Conclusion: In all the groups tested, clinically unacceptable $\Delta \mathrm{E}$ values were obtained. Although color stability of methacrylatebased composites has been widely investigated, this has not been done before with these kinds of immersion solutions.

Clinical significance: Potential discoloration might be limited by dietary adjustments based on in vitro evaluations.

\footnotetext{
${ }^{1-3}$ Department of Restorative Dentistry, Faculty of Dentistry Kirikkale University, Kırıkkale, Turkey

${ }^{4}$ Department of Busines Administartion, Faculty of Economics and Administrative Sciences, Abant Izzet Baysal University Bolu, Turkey

Corresponding Author: Erol Keskin, Department of Restorative Dentistry, Faculty of Dentistry, Kirikkale University, Kırıkkale Turkey, Phone: +913182244927, e-mail: dt.erolkeskin@ hotmail.com
}

Keywords: Color stability, Composite resins, Staining beverages.

How to cite this article: Baglar S, Keskin E, Orun T, Es A. Discoloration Effects of Traditional Turkish Beverages on different Composite Restoratives. J Contemp Dent Pract 2017;18(2): 83-93.

\section{Source of support: Nil}

\section{Conflict of interest: None}

\section{INTRODUCTION}

Due to the increasing demand, resin-based composites have undergone a significant evolution over the last decades, regarding both physicomechanical and esthetic properties. ${ }^{1,2}$ Thus, as composite resins (CRs) have the capability of remodeling the shape, recovering the function of the tooth, and maintaining the natural tooth appearance, and also being conservative because of their adhesion ability, their use both in anterior and posterior regions is fairly increasing. ${ }^{3-6}$ Considering these reasons, CRs are being employed as significant alternatives to ceramic veneers and porcelain-fused-to-metal crown restorations when esthetics are on the forefront. ${ }^{7}$ To provide the esthetics of dental restorations, especially, at the anterior region is still one of the greatest challenges in clinical practice. ${ }^{8}$ Regarding esthetics, the color stability of the restorative materials is one of the most important factors, which may cause the replacement of the restoration and lead to waste of extra time and money.

In the oral cavity, there are several factors, such as drinking habits, humidity, temperature changes, and hygiene routine that influence the CRs' susceptibility to discoloration. ${ }^{9-11}$ Discoloration of CRs may occur due to intrinsic discoloration or extrinsic staining or both. Intrinsic discoloration occurs depending on the resin matrix composition, initiator systems, conversion rate of matrix monomers, oxidation of the unreacted carbon double bonds, and the fillers' particle size and distribution. On the contrary, extrinsic staining occurs because of 
inadequate matrix polymerization, ultraviolet irradiation, water sorption, inadequate surface finishing, and adsorption of dietary colorants, such as coffee, tea, coke, fruit juices, and red wine. ${ }^{11-15}$

The drinks, above mentioned, are common in today's diet, and it has been proven by a lot of researches that some of them have the potential to stain resin composite materials. ${ }^{9-15}$ However, there are some different kinds of beverages, such as traditional ones, which may have probable staining potential. Tamarind syrup, ottoman syrup, and turnip juice are some traditional Turkish beverages. These drinks have originated from the Ottoman cuisine and have been formed as a result of a mixture of various plants and spices. ${ }^{14-18}$

There are very few reports in the literature about the discoloration effect of these kinds of materials on CRs. In their study, Stober et $\mathrm{al}^{19}$ reported that $0.1 \%$ turmeric solution caused discoloration on resin composites, and Imamura et $\mathrm{al}^{20}$ in 2008, highlighted the staining effect of curry on resin composites. More recently, Subramanya and Muttagi ${ }^{21}$ investigated the color change of dental veneering resins in tea, coffee, and tamarind extracts and stated that all materials including tamarind caused discoloration. Furthermore, Yew et al, ${ }^{14}$ evaluated the color stability of restorative resin composites upon exposure to spices of turmeric, paprika, and tamarind ( $0.1 \%$ weight solution), and they stated that all the tested spices showed the potential of staining, but turmeric showed the most significant discoloration. Therefore, although the color stability of methacrylate-based CRs immersed in staining solutions has been widely investigated, ${ }^{22-24}$ there are no in vitro studies, which evaluated the staining potential of these kinds of beverages.

Hence, the purpose of this study was to determine the staining potentials of tamarind syrup, ottoman syrup, turnip juice, and, in addition, pomegranate juice on different methacrylate-based CRs using a spectrophotometer device based on the CIE L*a* $b^{*}$ color system. Moreover, there were two null hypotheses of this study:

- All the selected solutions have the potential of staining the CRs.

- There is no significant difference in discoloration among different composite types.

\section{MATERIALS AND METHODS}

A total of 175 disk samples were prepared from five different CR materials (Table 1$)$ of shade A2 $(\mathrm{N}=35, \mathrm{n}=7)$ by condensing the resins into standardized Teflon molds. Molds were held between two glass slides, which were covered with transparent polyester strips (Mylar, DuPont,

Table 1: The composition of composite material used in this study

\begin{tabular}{|c|c|c|c|c|c|c|c|}
\hline Material & Type & Content & Filler content & $\begin{array}{l}\% \text { weight } \\
\text { and volume }\end{array}$ & $\begin{array}{l}\text { Lot } \\
\text { number }\end{array}$ & Shade & Manufacturer \\
\hline $\begin{array}{l}\text { Filtek ultimate } \\
\text { universal }\end{array}$ & $\begin{array}{l}\text { Nanofill } \\
\text { composite } \\
\text { nanocluster }\end{array}$ & $\begin{array}{l}\text { Bis-GMA, UDMA, triethylene } \\
\text { glycol dimethacrylate } \\
\text { (TEGDMA), poly (ethylene } \\
\text { glycol) dimethacrylate } \\
\text { (PEGDMA), ethoxylated } \\
\text { bisphenol-A dimethacrylate } \\
\text { (Bis-EMA) }\end{array}$ & $\begin{array}{l}20 \mathrm{~nm} \text { silica particles, } \\
4-11 \mathrm{~nm} \text { zirconium } \\
\text { particles }\end{array}$ & $\begin{array}{l}72.5 \text { wt } \% \\
55.6 \text { vol\% }\end{array}$ & N314606 & A2 & $\begin{array}{l}\text { 3M ESPE, } \\
\text { St. Paul, MN, } \\
\text { USA }\end{array}$ \\
\hline $\begin{array}{l}\text { Tetric } \\
\text { EvoCeram }\end{array}$ & Nanofill & $\begin{array}{l}\text { DMA, additives, catalysts, } \\
\text { stabilizers, pigments }\end{array}$ & $\begin{array}{l}\text { The fillers contain } \\
\text { barium glass, } \\
\text { ytterbium trifluoride, } \\
\text { mixed oxide and } \\
\text { prepolymer }\end{array}$ & $\begin{array}{l}75-76 \% \\
\text { weight, } \\
53-55 \% \\
\text { volume }\end{array}$ & L24180 & A2 & $\begin{array}{l}\text { Ivoclar } \\
\text { Vivadent } \\
\text { AG Schaan, } \\
\text { Liechtenstein }\end{array}$ \\
\hline $\begin{array}{l}\text { Clearfil } \\
\text { Majesty } \\
\text { Esthetic-2 } \\
\text { (enamel) }\end{array}$ & Nanofill & $\begin{array}{l}\text { Matrix: Bis-GMA, } \\
\text { hydrophobic aromatic } \\
\text { dimethacrylate, dl- } \\
\text { camphorquinone }\end{array}$ & $\begin{array}{l}\text { Silanated barium glass } \\
\text { and prepolymerized } \\
\text { organic filler, the } \\
\text { particle size of } \\
\text { inorganic fillers ranges } \\
\text { from } 0.37 \text { to } 1.5 \mu \mathrm{m}\end{array}$ & $\begin{array}{l}78 \text { wt } \% 40 \\
\text { vol\% }\end{array}$ & 00007C & A2 & $\begin{array}{l}\text { Kuraray Inc., } \\
\text { Tokyo, Japan }\end{array}$ \\
\hline $\begin{array}{l}\text { Clearfil } \\
\text { Majesty }^{\mathrm{TM}} \\
\text { Posterior }\end{array}$ & $\begin{array}{l}\text { Nano- } \\
\text { superfill }\end{array}$ & $\begin{array}{l}\text { Bis-GMA, triethylene } \\
\text { glycol dimethacrylate } \\
\text { (TEGDMA), aromatic } \\
\text { dimethacrylate (ArDMA), } \\
\text { dl-camphorquinone }\end{array}$ & $\begin{array}{l}\text { Silanated glass } \\
\text { ceramics, surface- } \\
\text { treated alumina, } \\
\text { microfiller, the particle } \\
\text { size of inorganic fillers } \\
\text { ranges from } 0.02 \text { to } \\
7.9 \mu \mathrm{m}\end{array}$ & $\begin{array}{l}92 \text { wt } \%, 82 \\
\text { vol\% }\end{array}$ & 4A0019 & A2 & $\begin{array}{l}\text { Kuraray Inc., } \\
\text { Tokyo, Japan }\end{array}$ \\
\hline $\begin{array}{l}\text { Quadrant } \\
\text { Universal LC }\end{array}$ & Nanofill & $\begin{array}{l}\text { Bis-GMA, triethylene glycol } \\
\text { dimethacrylate (TEGDMA) }\end{array}$ & $\begin{array}{l}\text { Ba-Al-F-silicate glass } \\
(0.02-2 \mu \mathrm{m}), \text { highly } \\
\text { dispersed silicon } \\
\text { dioxide }(0.02-0.07 \mu \mathrm{m})\end{array}$ & $\begin{array}{l}72 \text { wt } \%, 60 \\
\text { vol\% }\end{array}$ & 010637A & $\mathrm{A} 2$ & $\begin{array}{l}\text { Cavex } \\
\text { Haarlem, The } \\
\text { Netherlands }\end{array}$ \\
\hline
\end{tabular}


Wilmington, DE, USA). Slides were gently pressed and excess resin removed. Specimens were polymerized by a light-emitting diode (LED) curing unit (Elipar S10; 3M Unitek, Monrovia, CA, USA) with light intensity of $1200 \mathrm{~mW} / \mathrm{cm}^{2}$ for 20s both from top and bottom surfaces respectively, at a fixed distance of $1 \mathrm{~mm}$. Irradiance was tested by a radiometer Demetron LED (Kerr Corp, Orange, CA, USA). Specimens' dimensions were $10 \mathrm{~mm}$ in diameter and $2 \mathrm{~mm}$ thick. After polymerization, upper surfaces of specimens were polished with 400,800 , and 1,200 grit silicon carbide papers. Then, for rehydration and completion of the polymerization, all specimens were stored in distilled water at $37^{\circ} \mathrm{C}$ for 24 hours. ${ }^{14}$ Specimens of each CR were randomly divided into five subgroups corresponding to seven samples per staining solution. Then, specimens were rinsed with distilled water and dried with gauze before being immersed in staining solutions and distilled water as control. The $\mathrm{pH}$ of each solution was measured by a pH meter (SevenMulti S47, Mettler-Toledo AG, Schwerzenbach, Switzerland). The composition and $\mathrm{pH}$ of the solutions are shown in Table 2 .

Baseline color measurements (T0) were made before immersion. These measurements were performed using a VITA Easyshade Advance (Vident, Brea, CA) spectrophotometer device according to CIE L*a* $\mathrm{b}^{*}$ system against a white background. ${ }^{7}$ Randomly selected specimens from each material were immersed in $20 \mathrm{~mL}$ of the treatment solutions, which were kept in closed containers. Before each measurement session, the spectrophotometer was calibrated according to manufacturer's recommendations. Color measurements were made at a time interval of 3 days (T1), 6 days (T2), and 12 days (T3) according to the same procedure. Staining solutions were renewed every day to prevent microbial contamination.

Differences in color $(\Delta \mathrm{E})$ and color coordinates $\left(\Delta \mathrm{L}^{*}\right.$, $\Delta \mathrm{a}^{*}, \Delta \mathrm{b}^{*}$ ) between baseline (T0) and T1, T2, and T3 measurements were calculated for each resin-based composite material and staining solution. The $\Delta \mathrm{L}^{*}$ represents the change in luminosity, $\Delta \mathrm{a}^{*}$ represents the change in red-green parameter, and $\Delta \mathrm{b}^{*}$ represents the change in yellow-blue parameter. Color stability was determined to evaluate the color difference $(\Delta \mathrm{E})$. The $\Delta \mathrm{E}$ was calculated from the mean $\Delta \mathrm{L}^{*} \Delta \mathrm{a}^{*}$ and $\Delta \mathrm{b}^{*}$ according to the formula of $\Delta \mathrm{E}=\left[(\Delta \mathrm{L})^{2}+(\Delta \mathrm{a})^{2}+(\Delta \mathrm{b})^{2}\right]^{1 / 2} \cdot 1,3,6-9$

As data were normally distributed (KolmogorovSmirnov, Shapiro-Wilk), two-way analysis of variance was applied to evaluate and compare the effects of different materials and solutions on color changes regarding differences in $\Delta \mathrm{E}, \Delta \mathrm{L}, \Delta \mathrm{a}$, and $\Delta \mathrm{b}$. Furthermore, Tukey's honestly significant difference was used as post hoc test to evaluate data value of first and second time period in each group. The data analysis was performed using the Statistical Package for the Social Sciences version 19.0 (Inc, Chicago, Illinois, USA) with a significance set at $\mathrm{p}<0.05$ or less.

\section{RESULTS}

The means and standard deviations of $\Delta \mathrm{E}$ values at $\mathrm{T} 1$, $\mathrm{T} 2$, and $\mathrm{T} 3$ time intervals are shown in Table 3. At the end of the T1 period, the color change values $(\Delta \mathrm{E})$ varied between 2.83 and 6.93 (control group: 0.3-1.09). Clinically acceptable $\Delta \mathrm{E}$ limits in all solutions except control group are shown in Table 4.

The mean values of color change of the evaluated materials immersed in staining solutions at $\mathrm{T} 1$ to $\mathrm{T} 0$ period are represented in Table 3. Regarding the stainability potential, Clearfil Majesty ES-2 immersed in turnip juice showed the lowest color stability overall in the first 3 days, while the highest color stability was by Cavex Quadrant Universal LC immersed in tamarind syrup. Regarding the staining potential, turnip juice had the highest staining potential overall. The statistical analyses revealed after $\mathrm{T} 1$ to $\mathrm{T} 0$ period are shown in Tables 5 and 6.

For T2 period, the behavior of the materials and colorants is represented in Table 3. Clearfil Majesty ES-2 immersed in pomegranate juice showed the lowest color stability overall, while the highest color stability was by Clearfil Majesty Posterior immersed in Ottoman syrup. Regarding the staining potential, turnip juice showed the highest potential overall. The statistical analyses revealed after the $\mathrm{T} 2$ to $\mathrm{T} 0$ period are shown in Tables 7 and 8.

Table 2: Description of solutions

\begin{tabular}{|c|c|c|c|}
\hline Name & Composition & $\mathrm{pH}$ & Manufacturer \\
\hline $\begin{array}{l}\text { Tamarind syrup } \\
\text { (Tamarindus indica) }\end{array}$ & $\begin{array}{l}\text { Tamarind, water, sugar (powder sugar), orange peel, various spices } \\
\text { (ginger root, turmeric root, cinnamon stick, cloves, allspice, rendele } \\
\text { nutmeg, cardamom seed, rose water, fennel, anise) }\end{array}$ & 3.27 & $\begin{array}{l}\text { Rifat Minare canning factory, } \\
\text { Bursa, Turkey }\end{array}$ \\
\hline Ottoman syrup & $\begin{array}{l}\text { Water, honey, grape juice concentrate, pomegranate juice, lemon } \\
\text { juice concentrate, various spices (tamarind, cinnamon, ginger, } \\
\text { clove) }\end{array}$ & 3.06 & $\begin{array}{l}\text { AOÇ Commercial Outlet, } \\
\text { Ankara, Turkey }\end{array}$ \\
\hline $\begin{array}{l}\text { Turnip juice (Brassica } \\
\text { napobrassica) }\end{array}$ & $\begin{array}{l}\text { Fermented hot black carrot juice, water black carrot, salt, boiled and } \\
\text { pounded wheat, turnip, servative (sodium benzoate) }\end{array}$ & 3.59 & $\begin{array}{l}\text { AOÇ Commercial Outlet, } \\
\text { Ankara, Turkey }\end{array}$ \\
\hline $\begin{array}{l}\text { Pomegranate juice } \\
\text { (Punica granatum) }\end{array}$ & $\begin{array}{l}\text { Water, pomegranate juice concentrate (the amount of fruit juice } \\
\% 100 \text { ) }\end{array}$ & 3.24 & Doğanay Gıda, Adana, Turkey \\
\hline
\end{tabular}


Table 3: Mean value and standard deviation for $\Delta \mathrm{E}^{*}$ in different groups after 3, 6, and 12 days

\begin{tabular}{|c|c|c|c|c|c|c|}
\hline \multicolumn{7}{|c|}{ Color difference $\left(\Delta E^{*}\right)$} \\
\hline \multirow[b]{2}{*}{ Solution } & \multirow[b]{2}{*}{ Time } & $\begin{array}{l}\text { Filtek ultimate } \\
\text { universal }\end{array}$ & $\begin{array}{l}\text { Clearfil Majesty } \\
\text { ES-2 }\end{array}$ & $\begin{array}{l}\text { Quadrant } \\
\text { Universal LC }\end{array}$ & $\begin{array}{l}\text { Tetric } \\
\text { EvoCeram }\end{array}$ & $\begin{array}{l}\text { Clearfil Majesty } \\
\text { Posterior }\end{array}$ \\
\hline & & Mean (SD) & Mean (SD) & Mean (SD) & Mean (SD) & Mean (SD) \\
\hline \multirow[t]{3}{*}{ Distilled water } & After 3 days & $0.3(0.17)$ & $1.09(1)$ & $0.55(0.06)$ & $1.01(0.41)$ & $1.03(0.58)$ \\
\hline & After 6 days & $0.99(0.61)$ & $1.45(1.32)$ & $1.2(0.82)$ & $1.08(0.24)$ & $1.2(0.49)$ \\
\hline & After 12 days & $1.04(0.55)$ & $1.55(1)$ & $1.27(0.59)$ & $0.93(0.41)$ & $1.67(0.62)$ \\
\hline \multirow[t]{3}{*}{ Pomegranate juice } & After 3 days & $3.74(0.87)$ & $6.17(1.28)$ & $3.51(0.84)$ & $3.66(0.41)$ & $4.09(0.81)$ \\
\hline & After 6 days & $7.04(1.91)$ & $11.03(0.44)$ & $6.57(1.24)$ & $5.75(0.80)$ & $7.10(1.47)$ \\
\hline & After 12 days & $8.82(3.27)$ & $13.13(1.37)$ & $8.42(1.30)$ & $6.17(1.45)$ & $9.25(2.22)$ \\
\hline \multirow[t]{3}{*}{ Ottoman syrup } & After 3 days & $4.13(1.27)$ & $3.02(0.58)$ & $2.97(1.81)$ & $3.49(0.82)$ & $3.55(1.95)$ \\
\hline & After 6 days & $7.36(1.88)$ & $5.63(2.25)$ & $4.75(1.38)$ & $6.80(1.26)$ & $4.27(1.82)$ \\
\hline & After 12 days & $10.44(2.15)$ & $8.48(2.40)$ & $7.06(1.83)$ & $9.41(1.61)$ & $6.11(1.74)$ \\
\hline \multirow[t]{3}{*}{ Tamarind syrup } & After 3 days & $4.80(0.83)$ & $4.07(1.13)$ & $2.83(1.17)$ & $3.07(1.42)$ & $4.91(0.42)$ \\
\hline & After 6 days & $8.17(1.77)$ & $7.74(1.37)$ & $5.13(1.12)$ & $4.90(1.03)$ & $9.41(1.36)$ \\
\hline & After 12 days & $10.91(2.33)$ & $9.59(0.96)$ & $7.15(1.48)$ & $6.10(0.71)$ & $12.13(1.42)$ \\
\hline \multirow[t]{3}{*}{ Turnip juice } & After 3 days & $4.27(1.25)$ & $6.93(3.57)$ & $3.9(1.64)$ & $3.18(2.26)$ & $4.30(2.43)$ \\
\hline & After 6 days & $7.74(2.1)$ & $10.22(3.44)$ & $6.83(1.89)$ & $5.23(1.87)$ & $8.80(2.85)$ \\
\hline & After 12 days & 10.07 (3.69) & $11.17(3.74)$ & $8.81(1.90)$ & $6.24(2.17)$ & $12.41(2.51)$ \\
\hline
\end{tabular}

Table 4: Clinical acceptability values of the color change in the solution of composite materials in the range of T1 to T0

\begin{tabular}{lll}
\hline Composites & $T 1-T 0, \Delta E<3.3$ & $T 1-T 0, \Delta E>3.3$ \\
\hline Filtek Ultimate Universal & Distilled water $(0.3 \pm 0.17)$ & Turnip juice $(4.27 \pm 1.25)$ \\
& & Tamarind syrup $(4.80 \pm 0.83)$ \\
Clearfil Majesty ES-2 & & Ottoman syrup $(4.13 \pm 1.27)$ \\
& Distilled water $(1.09 \pm 1)$ & Pomegranate juice $(3.74 \pm 0.87)$ \\
Tetric EvoCeram & Ottoman syrup $(3.02 \pm 0.58)$ & Turnip juice $(6.93 \pm 3.57)$ \\
& & Tamarind syrup $(4.07 \pm 1.13)$ \\
Quadrant Universal LC & Distilled water $(1.01 \pm 0.41)$ & Pomegranate juice $(6.17 \pm 1.28)$ \\
& Turnip juice $(3.18 \pm 2.26)$ & Ottoman syrup $(3.49 \pm 0.82)$ \\
Clearfil Majesty Posterior & Tamarind syrup $(3.07 \pm 1.42)$ & Pomegranate juice $(3.66 \pm 0.41)$ \\
& Distilled water $(0.55 \pm 0.06)$ & \\
& Tamarind syrup $(2.83 \pm 1.17)$ & Turnip juice $(3.90 \pm 1.64)$ \\
& Ottoman syrup $(2.97 \pm 1.81)$ & Pomegranate juice $(3.51 \pm 0.84)$ \\
& Distilled water $(1.03 \pm 0.58)$ & Turnip juice $(4.30 \pm 2.43)$ \\
& & Tamarind syrup $(4.91 \pm 0.42)$ \\
& & Ottoman syrup $(3.55 \pm 1.95)$ \\
& & Pomegranate juice $(4.09 \pm 0.81)$
\end{tabular}

The behavior of the materials and colorants for the T3 period are represented in Table 3. Regarding the stainability potential, Clearfil Majesty ES-2 immersed in pomegranate juice showed the lowest color stability overall while the highest color stability was Tetric EvoCeram immersed in tamarind syrup. Regarding the staining potential, turnip juice showed the highest staining potential overall. The statistical analyses revealed after T3 to T0 period are shown in Tables 9 and 10.

\section{DISCUSSION}

Since dental treatments are costly and time-consuming processes, their longevity is desirable. In oral environmental situations, restorative materials are continuously exposed to water, hot or cold drinks, and saliva. ${ }^{25-28}$ These not only physically, but also visually affect the restorative materials. In this context, regarding color stability and staining resistance, it is very important to detect and know the behavior of the restorative materials used. $3,7,28$ In this present study, specimens were stored in staining solutions at $37^{\circ} \mathrm{C}$ in the dark for 12 days totally. A 24 hours of staining in vitro corresponds to 1 month in vivo, so this storage time was chosen to simulate the 1-year oral examination. ${ }^{9,28}$

According to the results of the present study, overall in all storage media, all the evaluated resin composites showed clinically unacceptable discolorations except T1 period. In this interval, resin composites of Tetric EvoCeram in turnip 
Table 5: Multiple comparison results ( $\mathrm{p}$ value) for the effect of composite type on the $\Delta \mathrm{E}$ in different storage media

\begin{tabular}{|c|c|c|c|c|c|}
\hline & $\begin{array}{l}\text { Filtek Ultimate } \\
\text { Universal }\end{array}$ & $\begin{array}{l}\text { Quadrant } \\
\text { Universal LC }\end{array}$ & $\begin{array}{l}\text { Clearfil Majesty } \\
\text { Posterior }\end{array}$ & $\begin{array}{l}\text { Clearfil Majesty } \\
\text { ES-2 }\end{array}$ & $\begin{array}{l}\text { Tetric } \\
\text { EvoCeram }\end{array}$ \\
\hline \multicolumn{6}{|c|}{ Filtek Ultimate Universal } \\
\hline Pomegranate juice & & 0.996 & 0.981 & 0.008 & 1.000 \\
\hline Ottoman syrup & & 0.747 & 0.973 & 0.743 & 0.960 \\
\hline Tamarind syrup & & 0.108 & 0.998 & 0.834 & 0.189 \\
\hline Turnip juice & & 0.999 & 1.000 & 0.499 & 0.967 \\
\hline Distilled water & & 0.953 & 0.321 & 0.209 & 0.338 \\
\hline \multicolumn{6}{|c|}{ Quadrant Universal LC } \\
\hline Pomegranate juice & 0.996 & & 0.892 & 0.004 & 0.999 \\
\hline Ottoman syrup & 0.747 & & 0.972 & 1.000 & 0.982 \\
\hline Tamarind syrup & 0.108 & & 0.061 & 0.433 & 0.997 \\
\hline Turnip juice & 0.999 & & 0.999 & 0.375 & 0.993 \\
\hline Distilled water & 0.953 & & 0.710 & 0.565 & 0.731 \\
\hline \multicolumn{6}{|c|}{ Clearfil Majesty Posterior } \\
\hline Pomegranate juice & 0.981 & 0.892 & & 0.027 & 0.962 \\
\hline Ottoman syrup & 0.973 & 0.972 & & 0.976 & 1.000 \\
\hline Tamarind syrup & 0.998 & 0.061 & & 0.657 & 0.111 \\
\hline Turnip juice & 1.000 & 0.999 & & 0.510 & 0.964 \\
\hline Distilled water & 0.321 & 0.710 & & 1.000 & 1.000 \\
\hline \multicolumn{6}{|l|}{ Clearfil Majesty ES-2 } \\
\hline Pomegranate juice & 0.008 & 0.004 & 0.027 & & 0.007 \\
\hline Ottoman syrup & 0.743 & 1.000 & 0.976 & & 0.985 \\
\hline Tamarind syrup & 0.834 & 0.433 & 0.657 & & 0.632 \\
\hline Turnip juice & 0.499 & 0.375 & 0.510 & & 0.195 \\
\hline Distilled water & 0.209 & 0.565 & 1.000 & & 0.999 \\
\hline \multicolumn{6}{|l|}{ Tetric EvoCeram } \\
\hline Pomegranate juice & 1.000 & 0.999 & 0.962 & 0.007 & \\
\hline Ottoman syrup & 0.960 & 0.982 & 1.000 & 0.985 & \\
\hline Tamarind syrup & 0.189 & 0.997 & 0.111 & 0.632 & \\
\hline Turnip juice & 0.967 & 0.993 & 0.964 & 0.195 & \\
\hline Distilled water & 0.338 & 0.731 & 1.000 & 0.999 & \\
\hline
\end{tabular}

T1 to T0 (Tukey's test, $p<0.05$ is considered as significant)

Table 6: Multiple comparison for the effect of storage media on the $\Delta \mathrm{E}$ in different composites

\begin{tabular}{|c|c|c|c|c|c|}
\hline & Distilled water & Pomegranate juice & Ottoman syrup & Tamarind syrup & Turnip juice \\
\hline \multicolumn{6}{|l|}{ Distilled water } \\
\hline Filtek Ultimate Universal & 0 & 0.001 & 0 & 0 & 0 \\
\hline Quadrant Universal LC & & 0.034 & 0.103 & 0.135 & 0.015 \\
\hline Clearfil Majesty Posterior & & 0.066 & 0.162 & 0.012 & 0.045 \\
\hline Clearfil Majesty ES-2 & & 0.010 & 0.477 & 0.323 & 0.010 \\
\hline Tetric EvoCeram & & 0.061 & 0.087 & 0.192 & 0.157 \\
\hline \multicolumn{6}{|l|}{ Pomegranate juice } \\
\hline Filtek Ultimate Universal & 0.001 & & 0.971 & 0.488 & 0.918 \\
\hline Quadrant Universal LC & 0.034 & & 0.973 & 0.940 & 0.992 \\
\hline Clearfil Majesty Posterior & 0.066 & & 0.985 & 0.890 & 1.000 \\
\hline Clearfil Majesty ES-2 & 0.010 & & 0.218 & 0.341 & 1.000 \\
\hline Tetric EvoCeram & 0.061 & & 1.000 & 0.961 & 0.981 \\
\hline \multicolumn{6}{|l|}{ Ottoman syrup } \\
\hline Filtek Ultimate Universal & 0 & 0.971 & & 0.832 & 0.999 \\
\hline Quadrant Universal LC & 0.103 & 0.973 & & 1.000 & 0.837 \\
\hline Clearfil Majesty Posterior & 0.162 & 0.985 & & 0.624 & 0.949 \\
\hline Clearfil Majesty ES-2 & 0.477 & 0.218 & & 0.998 & 0.218 \\
\hline Tetric EvoCeram & 0.087 & 1.000 & & 0.989 & 0.997 \\
\hline \multicolumn{6}{|l|}{ Tamarind syrup } \\
\hline Filtek Ultimate Universal & 0 & 0.488 & 0.832 & & $\begin{array}{l}0.918 \\
\quad(\text { Cont'd)... }\end{array}$ \\
\hline
\end{tabular}


$\left(\right.$ Cont'd $^{\prime} \ldots$

\begin{tabular}{|c|c|c|c|c|c|}
\hline & Distilled water & Pomegranate juice & Ottoman syrup & Tamarind syrup & Turnip juice \\
\hline Quadrant Universal LC & 0.135 & 0.940 & 1.000 & & 0.758 \\
\hline Clearfil Majesty Posterior & 0.012 & 0.890 & 0.624 & & 0.952 \\
\hline Clearfil Majesty ES-2 & 0.323 & 0.341 & 0.998 & & 0.340 \\
\hline Tetric EvoCeram & 0.192 & 0.961 & 0.989 & & 1.000 \\
\hline \multicolumn{6}{|l|}{ Turnip juice } \\
\hline Filtek Ultimate Universal & 0 & 0.918 & 0.999 & 0.918 & \\
\hline Quadrant Universal LC & 0.015 & 0.992 & 0.837 & 1.000 & \\
\hline Clearfil Majesty Posterior & 0.045 & 1.000 & 0.949 & 0.952 & \\
\hline Clearfil Majesty ES-2 & 0.010 & 1.000 & 0.218 & 0.340 & \\
\hline Tetric EvoCeram & 0.157 & 0.981 & 0.997 & 1.000 & \\
\hline
\end{tabular}

T1 to T0 (Tukey's test, $p<0.05$ is considered as significant)

Table 7: Multiple comparison results ( $p$ value) for the effect of composite type on the $\Delta E$ in different storage media

\begin{tabular}{|c|c|c|c|c|c|}
\hline & $\begin{array}{l}\text { Filtek Ultimate } \\
\text { Universal }\end{array}$ & $\begin{array}{l}\text { Quadrant } \\
\text { Universal LC }\end{array}$ & $\begin{array}{l}\text { Clearfil Majesty } \\
\text { Posterior }\end{array}$ & $\begin{array}{l}\text { Clearfil Majesty } \\
\text { ES-2 }\end{array}$ & $\begin{array}{l}\text { Tetric } \\
\text { EvoCeram }\end{array}$ \\
\hline \multicolumn{6}{|c|}{ Filtek Ultimate Universal } \\
\hline Pomegranate juice & & 0.983 & 1.000 & 0.002 & 0.598 \\
\hline Ottoman syrup & & 0.281 & 0.152 & 0.609 & 0.991 \\
\hline Tamarind syrup & & 0.027 & 0.924 & 0.985 & 0.016 \\
\hline Turnip juice & & 0.985 & 0.974 & 0.598 & 0.632 \\
\hline Distilled water & & 0.993 & 0.994 & 0.883 & 1.000 \\
\hline \multicolumn{6}{|c|}{ Quadrant Universal LC } \\
\hline Pomegranate juice & 0.983 & & 0.974 & 0.001 & 0.879 \\
\hline Ottoman syrup & 0.281 & & 0.995 & 0.945 & 0.504 \\
\hline Tamarind syrup & 0.027 & & 0.005 & 0.050 & 0.999 \\
\hline Turnip juice & 0.985 & & 0.802 & 0.307 & 0.894 \\
\hline Distilled water & 0.993 & & 1.000 & 0.987 & 0.999 \\
\hline \multicolumn{6}{|c|}{ Clearfil Majesty Posterior } \\
\hline Pomegranate juice & 1.000 & 0.974 & & 0.002 & 0.559 \\
\hline Ottoman syrup & 0.152 & 0.995 & & 0.785 & 0.306 \\
\hline Tamarind syrup & 0.924 & 0.005 & & 0.660 & 0.003 \\
\hline Turnip juice & 0.974 & 0.802 & & 0.914 & 0.309 \\
\hline Distilled water & 0.994 & 1.000 & & 0.986 & 0.999 \\
\hline \multicolumn{6}{|l|}{ Clearfil Majesty ES-2 } \\
\hline Pomegranate juice & 0.002 & 0.001 & 0.002 & & 0 \\
\hline Ottoman syrup & 0.609 & 0.945 & 0.785 & & 0.861 \\
\hline Tamarind syrup & 0.985 & 0.050 & 0.660 & & 0.030 \\
\hline Turnip juice & 0.598 & 0.307 & 0.914 & & 0.062 \\
\hline Distilled water & 0.883 & 0.987 & 0.986 & & 0.939 \\
\hline \multicolumn{6}{|l|}{ Tetric EvoCeram } \\
\hline Pomegranate juice & 0.598 & 0.879 & 0.559 & 0 & \\
\hline Ottoman syrup & 0.991 & 0.504 & 0.306 & 0.861 & \\
\hline Tamarind syrup & 0.016 & 0.999 & 0.003 & 0.030 & \\
\hline Turnip juice & 0.632 & 0.894 & 0.309 & 0.062 & \\
\hline Distilled water & 1.000 & 0.999 & 0.999 & 0.939 & \\
\hline
\end{tabular}

T2 to T0 (Tukey's test, $p<0.05$ is considered as significant)

juice (3.18 \pm 2.26$)$ and tamarind syrup (3.07 \pm 1.42$)$, Cavex Quadrant Universal LC in tamarind syrup $(2.83 \pm 1.17)$ and ottoman syrup (2.97 \pm 1.81$)$ and Clearfil Majesty ES-2 only in ottoman syrup $(3.02 \pm 0.58)$ showed near the border, but acceptable $(\Delta \mathrm{E}<3.3)$ discoloration values. Also, there were significantly different color changes among composite types in groups (Tables 5 to 10).
Hence, the first hypotheses, "all the selected solutions have the potential of staining the CRs" is accepted, but the second, "there are no significant differences in discoloration among different composite types" is rejected.

Anusavice et $\mathrm{al}^{29}$ stated that instrumental colorimetric measurements can eliminate subjective errors. For this purpose, colorimetry and spectrophotometry techniques 
Table 8: Multiple comparison for the effect of storage media on the $\Delta \mathrm{E}$ in different composites

\begin{tabular}{|c|c|c|c|c|c|}
\hline & Distilled water & Pomegranate juice & Ottoman syrup & Tamarind syrup & Turnip juice \\
\hline \multicolumn{6}{|l|}{ Distilled water } \\
\hline Filtek Ultimate Universal & & 0.001 & 0.001 & 0 & 0 \\
\hline Quadrant Universal LC & & 0 & 0.012 & 0.006 & 0 \\
\hline Clearfil Majesty Posterior & & 0.002 & 0.132 & 0 & 0 \\
\hline Clearfil Majesty ES-2 & & 0 & 0.063 & 0.014 & 0 \\
\hline Tetric EvoCeram & & 0 & 0 & 0.003 & 0.001 \\
\hline \multicolumn{6}{|l|}{ Pomegranate juice } \\
\hline Filtek Ultimate Universal & 0.001 & & 0.999 & 0.866 & 0.973 \\
\hline Quadrant Universal LC & 0 & & 0.331 & 0.548 & 0.999 \\
\hline Clearfil Majesty Posterior & 0 & & 0.183 & 0.584 & 0.630 \\
\hline Clearfil Majesty ES-2 & 0 & & 0.038 & 0.156 & 0.961 \\
\hline Tetric EvoCeram & 0.002 & & 0.708 & 0.837 & 0.968 \\
\hline \multicolumn{6}{|l|}{ Ottoman syrup } \\
\hline Filtek Ultimate Universal & 0.001 & 0.999 & & 0.956 & 0.997 \\
\hline Quadrant Universal LC & 0.012 & 0.331 & & 0.994 & 0.220 \\
\hline Clearfil Majesty Posterior & 0.132 & 0.183 & & 0.012 & 0.014 \\
\hline Clearfil Majesty ES-2 & 0.063 & 0.038 & & 0.931 & 0.127 \\
\hline Tetric EvoCeram & 0 & 0.708 & & 0.196 & 0.355 \\
\hline \multicolumn{6}{|l|}{ Tamarind syrup } \\
\hline Filtek Ultimate Universal & 0 & 0.866 & 0.956 & & 0.996 \\
\hline Quadrant Universal LC & 0.006 & 0.548 & 0.994 & & 0.396 \\
\hline Clearfil Majesty Posterior & 0 & 0.584 & 0.012 & & 1.000 \\
\hline Clearfil Majesty ES-2 & 0.014 & 0.156 & 0.931 & & 0.419 \\
\hline Tetric EvoCeram & 0.003 & 0.837 & 0.196 & & 0.994 \\
\hline \multicolumn{6}{|l|}{ Turnip juice } \\
\hline Filtek Ultimate Universal & 0 & 0.973 & 0.997 & 0.996 & \\
\hline Quadrant Universal LC & 0 & 0.999 & 0.220 & 0.396 & \\
\hline Clearfil Majesty Posterior & 0 & 0.630 & 0.014 & 1.000 & \\
\hline Clearfil Majesty ES-2 & 0 & 0.961 & 0.127 & 0.419 & \\
\hline Tetric EvoCeram & 0.001 & 0.968 & 0.355 & 0.994 & \\
\hline
\end{tabular}

T2 to T0 (Tukey's test, $p<0.05$ is considered as significant)

have been reliably used in various dental studies. ${ }^{6-10} \mathrm{So}$, to avoid bias due to individual subjective evaluation of color change, a spectrophotometric device was used in this study that allows a quantitative color assessment. Color measurements of the samples were made with CIE $L^{*} a^{*} b^{*}$ system because this system is well suited for the determination of small color differences and has been previously used in a large number of studies. ${ }^{30,31}$ Quantitative measurements are made in $\mathrm{L}^{*} \mathrm{a}^{*} \mathrm{~b}^{*}$ parameters and color change difference is calculated as $\Delta \mathrm{E}$. Theoretically, if no color difference is detected after its exposure to the testing media (namely $\Delta \mathrm{E}^{*}=0$ ), it may be claimed that material is completely color stable. ${ }^{25,26}$ Since for the anterior vestibular restorations like composite veneers, the most relevant background is white, we also preferred to use white background in our study. ${ }^{32}$

Resin composites used in dental restorations underwent color change when exposed to various storage media. The often-used solutions in research studies are coffee, black tea, red wine, coke, energy drinks, and some kind of soft beverages. ${ }^{30,32}$ In this sense, our study's difference were the staining solutions, such as tamarind syrup, ottoman syrup, and turnip juice, which were traditional (Table 2). There has been no article that evaluated the staining potential of these beverages on dental resin composites. Only in a few studies, some spices' staining potential on dental resins was evaluated, but, in these studies, only $1 \%$ solutions ${ }^{14}$ or extracts were used, ${ }^{21}$ and no beverages were evaluated. Yew et $\mathrm{al}^{14}$ evaluated the discoloration of two contemporary composites on exposure to spices of $0.1 \%$ weight, i.e., turmeric, paprika, and tamarind, and they reported that turmeric significantly stained the resin composites, but tamarind slightly and only after 168 hours affected the color of the resin composites. In their study, Subramanya and Muttagi ${ }^{21}$ found that the water extract of tamarind $(600 \mathrm{~mL}$ water $/ 60 \mathrm{gm}$ 
Table 9: Multiple comparison results ( $p$ value) for the effect of composite type on the $\Delta E$ in different storage media

\begin{tabular}{|c|c|c|c|c|c|}
\hline & $\begin{array}{l}\text { Filtek Ultimate } \\
\text { Universal }\end{array}$ & $\begin{array}{l}\text { Clearfil } \\
\text { Majesty ES-2 }\end{array}$ & $\begin{array}{l}\text { Quadrant } \\
\text { Universal LC }\end{array}$ & $\begin{array}{l}\text { Tetric } \\
\text { EvoCeram }\end{array}$ & $\begin{array}{l}\text { Clearfil } \\
\text { Majesty } \\
\text { Posterior }\end{array}$ \\
\hline \multicolumn{6}{|c|}{ Filtek Ultimate Universal } \\
\hline Distilled water & & 0.707 & 0.982 & 0.999 & 0.537 \\
\hline Pomegranate juice & & 0.032 & 0.998 & 0.354 & 0.997 \\
\hline Ottoman syrup & & 0.567 & 0.144 & 0.940 & 0.028 \\
\hline Tamarind syrup & & 0.671 & 0.015 & 0.002 & 0.722 \\
\hline Turnip juice & & 0.973 & 0.964 & 0.315 & 0.710 \\
\hline \multicolumn{6}{|l|}{ Clearfil Majesty ES-2 } \\
\hline Distilled water & 0.707 & & 0.951 & 0.539 & 0.998 \\
\hline Pomegranate juice & 0.032 & & 0.017 & 0.001 & 0.043 \\
\hline Ottoman syrup & 0.567 & & 0.810 & 0.949 & 0.339 \\
\hline Tamarind syrup & 0.671 & & 0.138 & 0.018 & 0.087 \\
\hline Turnip juice & 0.973 & & 0.702 & 0.097 & 0.950 \\
\hline \multicolumn{6}{|c|}{ Quadrant Universal LC } \\
\hline Distilled water & 0.982 & 0.951 & & 0.925 & 0.851 \\
\hline Pomegranate juice & 0.998 & 0.017 & & 0.507 & 0.968 \\
\hline Ottoman syrup & 0.144 & 0.810 & & 0.452 & 0.946 \\
\hline Tamarind syrup & 0.015 & 0.138 & & 0.848 & 0.001 \\
\hline Turnip juice & 0.964 & 0.702 & & 0.677 & 0.325 \\
\hline \multicolumn{6}{|l|}{ Tetric EvoCeram } \\
\hline Distilled water & 0.999 & 0.539 & 0.925 & & 0.379 \\
\hline Pomegranate juice & 0.354 & 0.001 & 0.507 & & 0.182 \\
\hline Ottoman syrup & 0.940 & 0.949 & 0.452 & & 0.128 \\
\hline Tamarind syrup & 0.002 & 0.018 & 0.848 & & 0 \\
\hline Turnip juice & 0.315 & 0.097 & 0.677 & & 0.026 \\
\hline \multicolumn{6}{|c|}{ Clearfil Majesty Posterior } \\
\hline Distilled water & 0.537 & 0.998 & 0.851 & 0.379 & \\
\hline Pomegranate juice & 0.997 & 0.043 & 0.968 & 0.182 & \\
\hline Ottoman syrup & 0.028 & 0.339 & 0.946 & 0.128 & \\
\hline Tamarind syrup & 0.722 & 0.087 & 0.001 & 0 & \\
\hline Turnip juice & 0.710 & 0.950 & 0.325 & 0.026 & \\
\hline
\end{tabular}

T3 to T0 (Tukey's test, $p<0.05$ is considered as significant)

tamarind powder) significantly discolored the autopolymerized tooth-colored acrylic resin. In this study, tamarind concentration highly corresponded to Yew et al's, and the authors stated that secondary metabolites, such as tannins, tartaric acid, and phenols were the primarily responsible agents regarding discoloration. In our study, tamarind-containing beverages (ottoman and tamarind syrups) showed significant discoloration values in all groups except in the T1 interval only for two composites for each beverage (Ottoman with Cavex Quadrant Universal LC and Clearfil Majesty ES-2 and tamarind with Tetric EvoCeram and Cavex Quadrant Universal LC) (Table 4). It probably occurred because of the concentration of tamarind and, especially, other substances present in the beverages (Table 2).

Neamat et $\mathrm{al}^{33}$ reported that low $\mathrm{pH}$ levels of potentially colorant beverages soften the resin matrix and chemical erosion may occur thus, negatively affecting the integrity of the tooth-colored restorations surfaces. This degradation may cause more water absorption and accompanying discoloration. The $\mathrm{pH}$ values of all the beverages used in this study were at low levels (Table 2). Nezhadnasrollah et $\mathrm{al}^{34}$ evaluated the discoloration effects of different herbal teas on exposure to different kinds of resin composites. In a part of their study, the authors stated that the highest discoloration occurred in the samples immersed in Hibiscus tea, which had the minimum $\mathrm{pH}$ value of 2.75 . In a high possibility, since discoloration increased as time progressed, the low $\mathrm{pH}$ directly proportionally affected the discoloration in this present study.

Dimensions of filler particles, volume of fillers in matrix, depth of polymerization, concentration and type of activators, initiators and inhibitors, oxidation of the unreacted carbon-carbon double bonds, coloring agents in daily diet, and resin matrix of dental composites are directly related to their color stability. ${ }^{31}$ Various studies have reported that the matrix structure of resin composites has an important impact on discoloration. ${ }^{25-27}$ Namely, since urethane dimethacrylate (UDMA) does 
Table 10: Multiple comparison for the effect of storage media on the $\Delta \mathrm{E}$ in different composites

\begin{tabular}{|c|c|c|c|c|c|}
\hline & Distilled water & Pomegranate juice & Ottoman syrup & tamarind syrup & turnip juice \\
\hline \multicolumn{6}{|l|}{ Distilled water } \\
\hline Filtek Ultimate Universal & & 0 & 0 & 0 & 0 \\
\hline Clearfil Majesty ES-2 & & 0 & 0.001 & 0 & 0 \\
\hline Quadrant Universal LC & & 0 & 0 & 0 & 0 \\
\hline Tetric EvoCeram & & 0 & 0 & 0 & 0 \\
\hline Clearfil Majesty Posterior & & 0 & 0.006 & 0 & 0 \\
\hline \multicolumn{6}{|l|}{ Pomegranate juice } \\
\hline Filtek Ultimate Universal & 0 & & 0.762 & 0.566 & 0.889 \\
\hline Clearfil Majesty ES-2 & 0 & & 0.026 & 0.125 & 0.634 \\
\hline Quadrant Universal LC & 0 & & 0.500 & 0.563 & 0.990 \\
\hline Tetric EvoCeram & 0 & & 0.005 & 1.000 & 1.000 \\
\hline Clearfil Majesty Posterior & 0 & & 0.050 & 0.083 & 0.049 \\
\hline \multicolumn{6}{|l|}{ Ottoman syrup } \\
\hline Filtek Ultimate Universal & 0 & 0.762 & & 0.997 & 0.999 \\
\hline Clearfil Majesty ES-2 & 0.001 & 0.026 & & 0.928 & 0.338 \\
\hline Quadrant Universal LC & 0 & 0.500 & & 1.000 & 0.264 \\
\hline Tetric EvoCeram & 0 & 0.005 & & 0.004 & 0.006 \\
\hline Clearfil Majesty Posterior & 0.006 & 0.050 & & 0 & 0 \\
\hline \multicolumn{6}{|l|}{ Tamarind syrup } \\
\hline Filtek Ultimate Universal & 0 & 0.566 & 0.997 & & 0.973 \\
\hline Clearfil Majesty ES-2 & 0 & 0.125 & 0.928 & & 0.790 \\
\hline Quadrant Universal LC & 0 & 0.563 & 1.000 & & 0.311 \\
\hline Tetric EvoCeram & 0 & 1.000 & 0.004 & & 1.000 \\
\hline Clearfil Majesty Posterior & 0 & 0.083 & 0 & & 0.999 \\
\hline \multicolumn{6}{|l|}{ Turnip juice } \\
\hline Filtek Ultimate Universal & 0 & 0.889 & 0.999 & 0.973 & \\
\hline Clearfil Majesty ES-2 & 0 & 0.634 & 0.338 & 0.790 & \\
\hline Quadrant Universal LC & 0 & 0.990 & 0.264 & 0.311 & \\
\hline Tetric EvoCeram & 0 & 1.000 & 0.006 & 1.000 & \\
\hline Clearfil Majesty Posterior & 0 & 0.049 & 0 & 0.999 & \\
\hline
\end{tabular}

T3 to T0 (Tukey's test, $p<0.05$ is considered as significant)

not have hydroxyl (-OH) side groups, it seems to be more color-resistant than bisphenol A-glycidyl methacrylate (Bis-GMA) because of its low water absorption and solubility characteristics. ${ }^{8} \mathrm{~A}$ water-absorbing $\mathrm{CR}$ is also more likely to absorb water-soluble pigments in the diet, which may cause discoloration of restorations. ${ }^{23,35}$ In this study, all evaluated composites were methacrylate-based and nano-filled ones. In all evaluation periods, although it is commercially introduced as for the esthetic use, Clearfil Majesty ES-2 showed the lowest color stability as $\Delta \mathrm{E}: 6.93 \pm 3.57$ at $\mathrm{T} 1, \Delta \mathrm{E}: 11.03 \pm 0.44$ at $\mathrm{T} 2$, and $\Delta \mathrm{E}$ : $13.13 \pm 1.37$ at $\mathrm{T} 3$. In various studies, authors stated that UDMA-based resins compared with other methacrylate monomer-based ones are less willing to change their color due to low water absorption and low viscosity. ${ }^{6-8,10,23,35}$ Moreover, it is also stated that the volume of fillers is linearly associated with erosion resistance in that erosion may cause discoloration directly and/or due to discoloring agents. ${ }^{36}$ On the contrary, less filler volume means more monomer matrix; so, this leads to more water absorption and degradation, which may cause more discoloration. ${ }^{23}$ As another point, researchers stated that barium-glass particles may tend to be more water absorbing. ${ }^{37,38}$ When these factors are considered, we were forced to make decisions on the reasons of discoloration differences because all of the resin composites were Bis-GMA-based materials and also all of them were nanofilled and barium glass particle-containing dental composites. However, considering the \% volume of filler contents of tested materials, differences were present. Especially, the most colored resin composite, Clearfil Majesty ES-2 was the least filler containing one. Therefore, based on the other studies, $3,6-8,12,13$ it can be argued that the filler content rate may have a significant effect on the color stability of resin composites. On the contrary, Clearfil Majesty Posterior was the second-most colored resin at the end of the study. But in contradiction, this composite was also the most filler containing one. Probably, rate differences of dimethacrylate derivatives in the monomer matrix and the surface treatments of the filler particles have a role in the discoloring differences we achieved in our study. For this purpose, we decided to do further studies on the interactions of these kinds of solutions with different kinds of resin composites. 
It seems that $\Delta \mathrm{E}$ values depended on the material and the staining solution in which the material was immersed. From T0 to T3, $\Delta \mathrm{E}$ increased gradually for every material, for which the stainability is also timedependent. When compared between composite materials at 12 days period (T3-T0), Clearfil Majesty ES-2 $(13.13 \pm 1.37)$ showed the highest $\Delta \mathrm{E}$ value, which was the same as the first 3 days (T1-T0) period. At the end of the 12 days period, Tetric EvoCeram showed the least $\Delta$ E value $(6.10 \pm 0.71)$.

Within the limitations of the present study, it can be concluded that the drinking habits as the part of the daily nutrition of the patients must be considered when choosing CR materials, especially, on the esthetic zone. Hence, it can be claimed that to provide the satisfactory esthetic appearance of restorations for a longer period in the oral environment; some restrictions about their dietary habits may be introduced to patients or appropriate restorative material selection may be done in conformity with the dietary lifestyle.

Therefore, the drinking habits as a part of the daily diet must be understood by the clinician in anamnesis and must be considered when choosing the resin composite material, especially, for the anterior esthetic restorations. Also, restorative materials' composition, polymerization degree, and surface texture-like properties are important to provide for their longevity and success.

\section{REFERENCES}

1. Gaintantzopoulou M, Kakaboura A, Vougiouklakis G. Colour stability of tooth-coloured restorative materials. Eur J Prosthodont Restor Dent 2005 Jun;13(2):51-56.

2. Oztürk E, Hickel R, Bolay S, Ilie N. Micromechanical properties of veneer luting resins after curing through ceramics. Clin Oral Investig 2012 Feb;16(1):139-146.

3. Fontes ST, Fernández MR, de Moura CM, Meireles SS. Color stability of a nanofill composite: effect of different immersion media. J Appl Oral Sci 2009 Sep-Oct;17(5):388-391.

4. Venturini D, Cenci MS, Demarco FF, Camacho GB, Powers JM. Effect of polishing techniques and time on surface roughness, hardness and microleakage of resin composite restorations. Oper Dent 2006 Jan-Feb;31(1):11-17.

5. Da Costa J, Ferracane J, Paravina RD, Mazur RF, Roeder L. The effect of different polishing systems on surface roughness and gloss of various resin composites. J Esthet Restor Dent 2007;19(4):214-224.

6. Ardu S, Braut V, Gutemberg D, Krejci I, Dietschi D, Feilzer AJ. A long-term laboratory test on staining susceptibility of esthetic composite resin materials. Quintessence Int 2010 Sep;41(8):695-702.

7. Arocha MA, Mayoral JR, Lefever D, Mercade M, Basilio J, Roig M. Color stability of siloranes versus methacrylate-based composites after immersion in staining solutions. Clin Oral Investig 2013 Jul;17(6):1481-1487.

8. Arocha MA, Basilio J, Llopis J, Di Bella E, Roig M, Ardu S, Mayoral JR. Colour stainability of indirect CAD-CAM processed composites vs. conventionally laboratory processed composites after immersion in staining solutions. J Dent 2014 Jul;42(7):831-838.

9. Patel SB, Gordan VV, Barrett AA, Shen C. The effect of surface finishing and storage solutions on the color stability of resin-based composites. J Am Dent Assoc 2004 May;135(5): 587-594.

10. Falkensammer F, Arnetzl GV, Wildburger A, Freudenthaler J. Color stability of different composite resin materials. J Prosthet Dent 2013 Jun;109(6):378-383.

11. Bansal K, Acharya SR, Saraswathi V. Effect of alcoholic and non-alcoholic beverages on color stability and surface roughness of resin composites: an in vitro study. J Conserv Dent 2012 Jul;15(3):283-288.

12. Erdemir U, Yildiz E, Eren MM. Effects of sports drinks on color stability of nanofilled and microhybrid composites after long-term immersion. J Dent 2012 Dec;40(Suppl 2):e55-e63.

13. Moon JD, Seon EM, Son SA, Jung KH, Kwon YH, Park JK. Effect of immersion into solutions at various $\mathrm{pH}$ on the color stability of composite resins with different shades. Restor Dent Endod 2015 Nov;40(4):270-276.

14. Yew HZ, Berekally TL, Richards LC. A laboratory investigation of colour changes in two contemporary resin composites on exposure to spices. Aust Dent J 2013 Dec;58(4):468-477.

15. Sudjaroen Y, Haubner R, Würtele G, Hull WE, Erben G, Spiegelhalder B, Changbumrung S, Bartsch H, Owen RW. Isolation and structure elucidation of phenolic antioxidants from Tamarind (Tamarindus indica L.) Seeds and pericarp. Food Chem Toxicol 2005 Nov;43(11):1673-1682.

16. Rodriguez Amado JR, Lafourcade Prada A, Escalona ArranzJC, Pérez Rosés R, Morris Quevedo H, Keita H, Puente Zapata E, Pinho Fernandes C, Tavares Carvalho JC. Antioxidant and hepatoprotective activity of a new tablets formulation from Tamarindus indica L. Evid Based Complement Alternat Med 2016;2016:3918219.

17. Razali N, Abdul Aziz A, Lim CY, Mat Junit S. Investigation into the effects of antioxidant-rich extract of Tamarindus indica leaf on antioxidant enzyme activities, oxidative stress and gene expression profiles in HepG2 cells. PeerJ 2015 Oct;3:e1292.

18. Pasko P, Bukowska-Strakova K, Gdula-Argasinska J, TyszkaCzochara M. Rutabaga (Brassica napus L. var. napobrassica) seeds, roots, and sprouts: a novel kind of food with antioxidant properties and proapoptotic potential in Hep G2 hepatoma cell line. J Med Food 2013 Aug;16(8):749-759.

19. Stober T, Gilde H, Lenz P. Color stability of highly filled composite resin materials for facings. Dent Mater 2001 Jan;17(1):87-94.

20. Imamura S, Takahashi H, Hayakawa I, Loyaga-Rendon PG, Minakuchi S. Effect of filler type and polishing on the discoloration of composite resin artificial teeth. Dent Mater J 2008 Nov;27(6):802-808.

21. Subramanya JK, Muttagi S. In vitro color change of three dental veneering resins in tea, coffee and tamarind extracts. J Dent (Tehran) 2011 Summer;8(3):138-145.

22. Kolbeck C, Rosentritt M, Lang R, Handel G. Discoloration of facing and restorative composites by UV-irradiation and staining food. Dent Mater 2006 Jan;22(1):63-68.

23. Bagheri R, Burrow MF, Tyas M. Influence of food-simulating solutions and surface finish on susceptibility to staining of aesthetic restorative materials. J Dent 2005 May;33(5):389-398.

24. Topcu FT, Sahinkesen G, Yamanel K, Erdemir U, Oktay EA, Ersahan S. Influence of different drinks on the colour stability of dental resin composites. Eur J Dent 2009 Jan;3(1):50-56. 
25. Janda R, Roulet JF, Kaminsky M, Steffin G, Latta M. Color stability of resin matrix restorative materials as a function of the method of light activation. Eur J Oral Sci 2004 Jun;112(3):280-285.

26. Reis AF, Giannini M, Lovadino JR, Ambrosano GM. Effects of various finishing systems on the surface roughness and staining susceptibility of packable composite resins. Dent Mater 2003 Jan;19(1):12-18.

27. Türkün LS, Türkün M. Effect of bleaching and repolishing procedures on coffee and tea stain removal from three anterior composite veneering materials. J Esthet Restor Dent 2004;16(5):290-301.

28. Ertaş E, Güler AU, Yücel AC, Köprülü H, Güler E. Color stability of resin composites after immersion in different drinks. Dent Mater J 2006 Jun;25(2):371-376.

29. Anusavice, KJ. Phillips' science of dental materials. 11th ed. St. Louis: Elsevier; 2003. p. 46-51.

30. Setz J, Engel E. In vivo color stability of resin-veneered telescopic dentures: a double blind pilot study. J Prosthet Dent 1997 May;77(5):486-491.

31. Shintani H, Satou N, Yukihiro A, Satou J, Yamane I, Kouzai T, Andou T, Kai M, Hayashihara H, Inoue T. Water sorption, solubility and staining properties of microfilled resins polished by various methods. Dent Mater J 1985 Jun;4(1): 54-62.
32. Dietschi D, Olsburgh S, Krejci I, Davidson C. In vitro evaluation of marginal and internal adaptation after occlusal stressing of indirect class II composite restorations with different resinous bases. Eur J Oral Sci 2003 Feb;111(1):73-80.

33. Abu-Bakr N, Han L, Okamoto A, Iwaku M. Changes in the mechanical properties and surface texture of compomer immersed in various media. J Prosthet Dent 2000 Oct;84(4):444-452.

34. Nezhadnasrollah F, Shahrokhzadeh F, Gholinia H. Evaluation of the effect of different herbal tea on discoloration of different kinds of composite. J Dent Mater Tech 2016 May;5(3):59-62.

35. Kerby RE, Knobloch LA, Schricker S, Gregg B. Synthesis and evaluation of modified urethane dimethacrylate resins with reduced water sorption and solubility. Dent Mater 2009 Mar;25(3):302-313.

36. Han L, Okamoto A, Fukushima M, Okiji T. Evaluation of flowable resin composite surfaces eroded by acidic and alcoholic drinks. Dent Mater J 2008 May;27(3):455-465.

37. Hubbezoglu I, Akaoğlu B, Dogan A, Keskin S, Bolayir G, Ozçelik S, Dogan OM. Effect of bleaching on color change and refractive index of dental composite resins. Dent Mater J 2008 Jan;27(1):105-116.

38. Hirata R. Clinical alternative of laboratory composite resin systems - when and how to use. J Bras Clin Estet Odontol 2000;4:13-21. 